\title{
Decreased Superior Sagittal Sinus Diameter and Jugular Bulb Narrowing Are Associated with Poor Clinical Outcome in Vein of Galen Arteriovenous Malformation
}

\author{
(D) G. Saliou, DP. Dirks, DR.H. Sacho, (D) Chen, (D) K. terBrugge, and (D)T. Krings
}

\begin{abstract}
BACKGROUND AND PURPOSE: Few clinical and imaging findings are known to be associated with poor outcome in neonates and infants with vein of Galen arteriovenous malformations. In the present consecutive series of 35 patients, we evaluated both the diameter of the superior sagittal sinus at onset and the diameter of the jugular bulb on follow-up as potential factors related to poor outcome.
\end{abstract}

MATERIALS AND METHODS: Thirty-five consecutive neonates and infants who were prospectively collected in a single-center data base were included in this review. Outcome was assessed by using the Bicêtre Outcome Score. Both the absolute diameter of the superior sagittal sinus and its ratio to the biparietal diameter were measured at onset, compared with age-matched controls, and correlated to patient outcome.

RESULTS: The diameter of the superior sagittal sinus at onset and its ratio to the biparietal diameter were significantly smaller in the vein of Galen arteriovenous malformation population compared with the matched population $(P=.0001)$ and were correlated significantly with a risk of poor clinical outcome $(P=.008)$. Development of jugular bulb narrowing was also related to poor clinical outcome $(P<.0001)$.

CONCLUSIONS: Decreased superior sagittal sinus diameter may reflect a decrease of cerebral blood flow due to cerebral arterial steal and intracranial hydrovenous disorders. This finding may be considered cerebral blood flow deterioration and thus taken into consideration in the management decisions for patients with vein of Galen arteriovenous malformations. Likewise, our data suggest that progressive jugular bulb narrowing may indicate earlier intervention to prevent severe narrowing.

ABBREVIATIONS: SSS = superior sagittal sinus; VGAM = vein of Galen arteriovenous malformation

V ein of Galen aneurysmal malformations are rare congenital choroidal arteriovenous malformations. ${ }^{1}$ In true Vein of Galen arteriovenous malformations (VGAMs), arteriovenous fistulas drain into an aberrantly persistent fetal median prosencephalic vein, coined the "vein of Markowski," which constitutes an embryonic precursor of the vein of Galen, thus

Received May 28, 2015; accepted after revision December 16

From the Department of Neuroradiology (G.S., R.H.S., L.C., K.T., T.K.), Toronto Western Hospital and University Health Network, Toronto, Ontario, Canada; Department of Neuroradiologie (G.S.), Centre Hospitalier Universitaire Bicêtre, Le Kremlin-Bicêtre, France; and Division of Neurosurgery (P.D.), The Hospital for Sick Children, Toronto, Ontario, Canada.

Guillaume Saliou was responsible for conception and design, acquisition of data and drafting the article, statistical analysis, and analysis and interpretation of data. Peter Dirks was responsible for conception and design, acquisition of data and drafting the article, and analysis and interpretation of data. Raphael H. Sacho was responsible for acquisition of data and drafting the article and analysis and interpretation of data. Long Chen was responsible for statistical analysis, drafting the article, and analysis and interpretation of data. Karel terBrugge was responsible for acquisition of data and drafting the article and analysis and interpretation of data. Timo Krings was responsible for conception and design, acquisition of data and drafting the article, and analysis and interpretation of data.

Paper previously presented in part at: ABC-WIN Conference, January 18-23, 2015; Val d'Isère, France. forcing the deep venous system to drain through persistent alternative embryonic routes, mainly the lateromesencephalic and lateropontine veins and the superior petrosal sinus. ${ }^{3}$ VGAMs are supposed to be associated with high morbidity and mortality. ${ }^{4-8}$ Lasjaunias et $\mathrm{al}^{9,10}$ proposed a neonatal scoring system for these malformations, a multiorgan evaluation, to decide the indication and timing of treatment. Certain clinical and imaging findings, such as a low neonatal score, ${ }^{9,10,11}$ encephalomalacia, ${ }^{10-12}$ intraparenchymal calcifications, ${ }^{10}$ and the angioarchitecture of the VGAM (choroidal type nidus and jugular stenosis without cavernous drainage), have been associated with a poorer prognosis.

In the present series, we tried to determine additional signs identifiable on cross-sectional imaging that may be associated with poor clinical outcome. Anecdotally, we have observed that the diameter of the superior sagittal sinus (SSS) in babies with VGAM appeared smaller compared with children without cere-

Please address correspondence to Guillaume Saliou, MD, PhD, Department of Neuroradiology, Toronto Western Hospital and University Health Network, 3MCL429, 399 Bathurst St, Toronto, ON, M5T 2S8 Canada; e-mail address: guillaume.saliou@bct.aphp.fr

http://dx.doi.org/10.3174/ajnr.A4697 
bral arteriovenous malformations and that on angiography, a delayed and decreased cortical venous drainage through the SSS was present. We presumed that these findings were related to the downstream venous hypertension induced by the arteriovenous shunt; therefore, it could be an indirect marker for high-flow angiopathy, venous congestion, and arterial steal, which, in turn, may be associated with poor clinical outcome. Therefore, in the present series, we wanted to evaluate, in a consecutive series, whether a smaller diameter of the superior sagittal sinus, which can already be present at birth in babies with VGAM, can predict outcome. In addition, we evaluated whether jugular bulb stenosis or occlusion, which are more likely to appear during the first year of life, can predict outcome.

\section{MATERIALS AND METHODS Patient Population}

The patient cohort was extracted from a data base of vein of Galen malformations managed at a single center (The Hospital for Sick Children, Toronto, Canada). Thirty-five consecutive children with vein of Galen aneurysmal malformation were included in this study from January 2004 to June 2014 after institutional ethics board approval was obtained. Only "true" VGAMs were included (ie, pediatric patients with pial AVMs that drained into the vein of Galen were not included).

All patients who were treated with embolization were treated with intra-arterial $N$-butyl cyanoacrylate injection by the 2 senior authors with the patient under general anesthesia. Embolization was performed in an attempt to control heart failure or hydrodynamic disorder in the neonatal period or to enable normal psychomotor development as previously proposed., ${ }^{9,10}$

\section{Clinical Features}

We retrospectively reviewed the following clinical data: sex, scoring (Bicêtre neonatal score), ${ }^{9,10}$ and hemodynamic parameters at clinical presentation (cardiac failure and suprasystemic pulmonary hyperpressure). The follow-up outcome for all patients was assessed by using the Bicêtre Outcome Score (Appendix). ${ }^{9,10}$ Poor outcome was defined as a Bicêtre Outcome Score of 0 or 1 .

\section{Radiologic Features}

Imaging review included conventional MR imaging and digital subtraction angiography. For the purpose of this study, we focused on first imaging (onset) and follow-up MR imaging performed between 12 and 24 months of age.

The following angioarchitectural features were studied on DSA before embolization: type of VGAM (ie, mural or choroidal) and type of flow (high or low) and superior sagittal sinus reflux. A VGAM associated with cardiac failure was considered high-flow. The following features were studied on first MR imaging: superior sagittal sinus diameter and encephalomalacia. The measurement of the SSS diameter was performed by the same operator (G.S.), who had $>10$ years' experience in neuroradiology. The maximal transverse diameter was measured. If a cortical vein reached the sinus at this level, the measurements were performed in an adjacent region of the sinus without cortical veins, to avoid measurement errors. In vein of Galen malformation, the normal triangular shape of the sinus was often not present and was replaced with

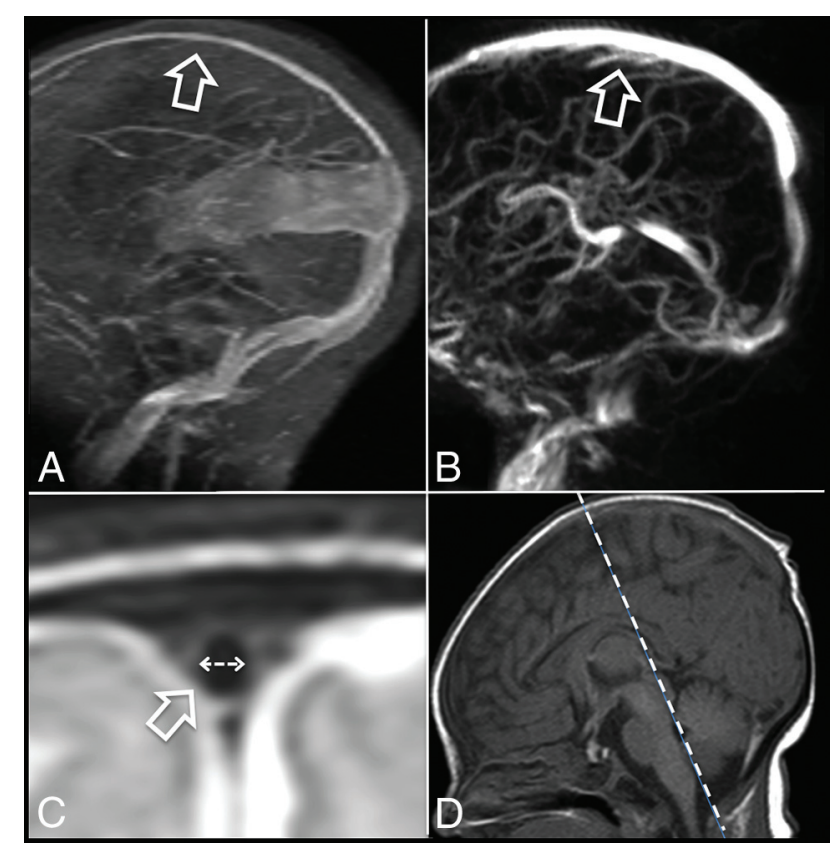

FIG 1. Decreased diameter of the superior sagittal sinus in a patient with a vein of Galen aneurysmal malformation at onset ( $A$, arrow) compared with an age-matched control $(B$, arrow). The width of the superior sagittal sinus was assessed on a T2-TSE-weighted sequence (flow void; $C$, arrow) in the coronal view crossing the posterior part of the brain stem $(D$, dotted line) and indicates significant reduction in the vein of Galen aneurysmal malformation.

an ovoid sinus. "Encephalomalacia" was defined as any focal or regional cerebral white and gray matter change with atrophy on imaging.

Because the size of the skull and every organ grows according to the infant's age, we related the SSS diameter, as measured on coronal T2-TSE sequences, to the biparietal diameter. Thus, the SSS index was calculated as the width of the flow void in the sagittal sinus on coronal T2 spin-echo/biparietal diameter $\times 100$. The SSS width was always assessed on a coronal view in a section passing through the tectum (Fig 1), and care was taken that in all cases, a similar coronal angulation was used. We also measured the SSS index in an age-matched population of 35 children without VGAM who underwent MR imaging for a reason other than vascular disease after institutional ethics board approval was obtained. The MRIs were performed for psychomotor delay (19 patients), seizure (11 patients), and suspicion of encephalitis (5 patients). All these MRI findings were considered normal. These patients were chosen because they were the same age as those in our population study: fewer than 5 days difference for the patients younger than 2 months of age; 15 days, for those younger than 6 months of age; 1 month, for those younger than 1 year of age; and 2 months, after 1 year of age. We considered that an SSS index inferior or equal to the 10th percentile of the matched population reflected a decrease in the SSS diameter.

The percentage of jugular bulb narrowing was assessed on CTA or DSA as the smallest diameter of the jugular bulb divided by the diameter of the jugular veins $2 \mathrm{~cm}$ below the jugular foramen (Fig 2). We considered bilateral narrowing of $>75 \%$ severe. Bilateral narrowing of $<75 \%$ or unilateral narrowing was not considered a severe narrowing.

AJNR Am J Neuroradiol 37:1354-58 Jul 2016 www.ajnr.org 1355 


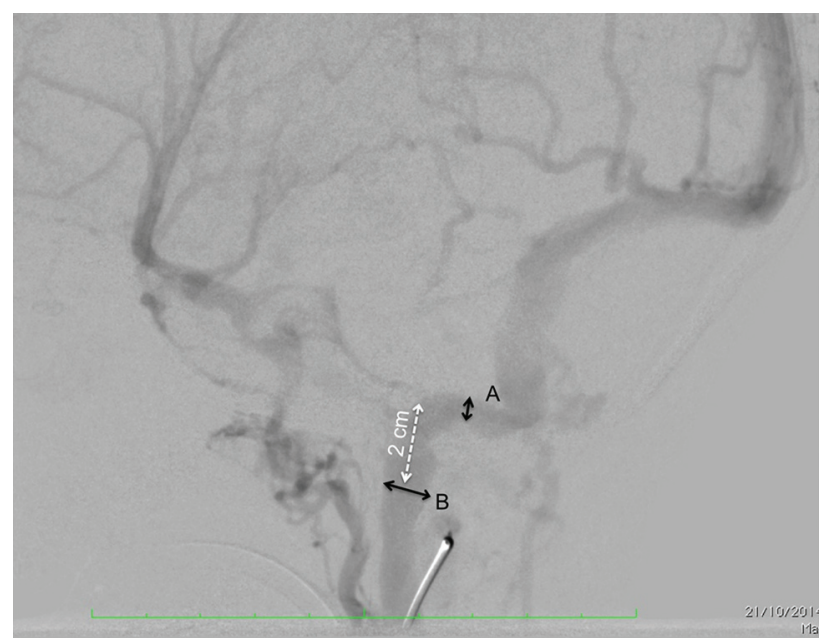

FIG 2. Method of assessment of jugular bulb stenosis at angiography. A corresponds to the maximal stenosis of the jugular bulb. B corresponds to the maximal diameter of the jugular vein at a point $2 \mathrm{~cm}$ (white dotted arrow) below its exit from the jugular foramina. The percentage of stenosis was calculated as A / B $\times 100$.

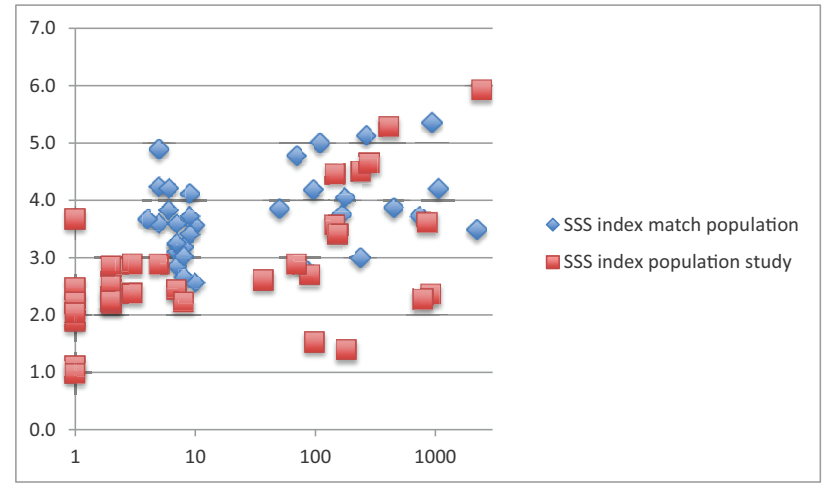

FIG 3. SSS index in the study population (red squares) and the matched population (blue diamonds) according to the age of the patient (days).

\section{Statistical Analysis}

Descriptive statistics were performed to summarize the distribution of clinical features across patients with and without decreased SSS diameter (Table). The distribution of categoric variables was described by frequencies and percentages; continuous and normally distributed variables, by means and SDs; and continuous and non-normally distributed variables, by medians and interquartile ranges. Univariate statistics were performed via $\chi^{2}$ or Fisher exact tests (comparison of categoric variables) to assess the associations among clinical features, SSS index, and jugular bulb stenosis. To evaluate whether the SSS index in VGAM was decreased due only to an increase of the biparietal diameter (for instance in case of hydrocephalus, frequently observed in VGAM), we also compared the absolute number of the diameter of the SSS in the VGAM population with the matched population, without taking into account the biparietal diameter. A Student $t$ test was performed to assess a difference between the population with a VGAM and the matched population regarding the SSS index. A $P$ value $<.05$ was statistically significant.

\section{RESULTS}

Between January 2000 and June 2014, 35 consecutive children were identified who fulfilled our inclusion criteria. We had a median clinical follow-up of 356 days (interquartile range, 10.5-1955 days). There were 12 (34\%) females and 23 (66\%) males. The median age at diagnosis was 5 days (interquartile range, 1.5-150.5 days). There was no statistical difference with the control group, in which the median age was 9 days (interquartile range, 7-136.5 days).

On the basis of the control group, we determined the 10th percentile of the SSS index to be 3. Consequently, we considered that an SSS index of $\leq 3$ indicated a decreased SSS diameter. Twenty-one (60\%) patients had an SSS index of $\leq 3$ on their first MR imaging. Overall, 6 patients had bilateral jugular bulb stenosis of $>75 \%$. However, only 1 of 20 patients who were scanned in their first month of life exhibited this bilateral stenosis, whereas in this subgroup of 20 patients, 16 already had an SSS index of $<3$.

The SSS index was significantly smaller in the VGAM population study group compared with the matched population $(P=$ .0001) (Fig 3). The comparison of the absolute numbers of the diameter of the SSS in the VGAM population with those in the matched population, without taking into account the biparietal diameter, also showed significant $(P=.03)$ differences (median, $2.35 \mathrm{~mm}$; interquartile range, $2-3 \mathrm{~mm}$; and median, $3.6 \mathrm{~mm}$; interquartile range, $2.9-4.45 \mathrm{~mm}$ in the VGAM group and matched population, respectively).

Factors associated with a risk of poor clinical outcome were presentation with cardiac failure $(P=.043)$, an SSS index of $\leq 3$ at onset $(P=.008)$, and presentation with encephalomalacia $(P=.004)$.

As only 1 child presented with bilateral jugular bulb narrowing, no statistical relevance was found between this feature and poor outcome at onset; however, bilateral jugular bulb stenosis at $1-2$ years of age was associated with poor clinical outcome $(P<.0001)$.

Main findings on statistical analysis are summarized in the Table.

\section{DISCUSSION}

Multiple factors have been associated with poor clinical outcome in babies with vein of Galen arteriovenous malformation. ${ }^{10-12}$ Among them, a Bicêtre neonatal score of $<12$, parenchymal changes or calcifications as a sign of manifest brain damage, arterial steal with visible diffusion abnormalities before treatment, cardiac failure, and multiorgan failure have been described. To our knowledge, this is the first series to report that decreased diameter of the SSS in VGAM at diagnosis and evolution of jugular bulb narrowing with time are also associated with poor clinical outcome.

In the present series, the overall population with a VGAM tended to have a smaller superior sagittal sinus diameter, and within the group of patients with VGAM, a decreased diameter was associated with poor clinical outcome. In addition, patients who were seen to develop jugular bulb narrowing also had an unfavorable clinical outcome.

Three aspects distinguish the jugular bulb narrowing from the SSS diameter narrowing: Unlike the focal jugular bulb narrowing, 


\section{Summary}

P Value

SSS index decreased at onset (ie, <10th percentile of matched population)

Superior sagittal sinus reflux on angiography at onset

Type of the vein of Galen malformation at onset (high-flow vs slow-flow)

Risk of bilateral jugular bulb stenosis $>75 \%$ at $1-2$ years

Angioarchitecture at onset

Bilateral jugular bulb stenosis at onset

Encephalomalacia at onset

Type of the vein of Galen malformation at onset (high-flow vs slow-flow)

Bilateral jugular bulb stenosis at 1-2 years on MRI

SSS index in the present series and matched population
Poor clinical outcome (last clinical assessment)

outcome in the initial imaging work-up of babies with VGAM. This can be of particular importance when contemplating a more urgent treatment to control cerebral blood flow and/or early hydrovenous disorders. Conversely, a normal SSS diameter may support delayed treatment.

Jugular bulb stenosis or occlusion has previously been called "dysmaturation of the jugular bulbs." 9 In our experience, this progressive narrowing is commonly observed in patients with VGAM. The influence of abnormal skull the decreased diameter of the SSS was noted along the entire length of the SSS. Second, while the SSS diameter decrease was typically present at onset, jugular bulb narrowing almost always developed later during the course of the disease. Third, while the arterialized blood flow from the VGAM shunt did use the jugular bulbs as an outflow pathway, it did not use the superior sagittal sinus (with the exception made for rare cases where SSS reflux was noted).

Although at present the cause and nature of the venous diameter reduction remain unclear, the above-mentioned considerations make it likely that different causes may be present. While there are no pathologically proved concepts of why the SSS diameter is decreased or why this is associated with poor clinical outcome, one may, however, propose the following hypotheses: First, the decreased diameter is related to decreased volume of venous flow and thus a collapse of the SSS. Because the SSS drains most cortical veins, the increase of intracranial pressure, the high-flow cardiac failure, and the arterial steal toward the shunt may lead to a decreased blood flow through the brain and thus into the sinuses. Second, an increase in intracranial pressure may be responsible for external compression of the SSS. Given these 2 hypotheses, SSS narrowing may be secondary to cerebral blood flow deterioration and thus explain why those patients had a worse outcome. Because patients with chronic hydrovenous disorders and high-flow cardiac failure ${ }^{10-12}$ are known to be at risk of psychomotor delay, the apparent narrowing of the SSS may therefore not be an independent predictor but rather an imaging correlate of the underlying pathologic mechanism. One may, however, presume that the SSS narrowing has already occurred early in the course of the disease and thus may represent an imaging correlate that indicates an early intervention. Thus, we believe that the described imaging findings may be an important decision-making tool in the discussion of the optimal treatment window.

Alternatively, the narrowing of the SSS may be related to a true stenosis, similar to the believed cause of the jugular bulb narrowing. This may be related to in utero reflux, though we have not observed this in this retrospective series. It is unlikely that both findings have the same underlying cause: Only a few patients had bilateral jugular bulb stenosis at onset (6 patients, 17\%). However, $66 \%$ already had decreased SSS diameter.

Therefore, we hypothesize that the decreased flow in the SSS was a secondary reflection of a combination of increased intracranial pressure, arterial steal, and low cardiac output. Thus, it may represent an important imaging correlate that may help predict base growth maturation caused by macrocrania as opposed to a venous high-flow angiopathy has been put forward as a potential cause. ${ }^{9,13}$ Jugular bulb narrowing may protect the heart by decreasing the venous pressure arising in the right heart. If the jugular bulb stenosis is severe, drainage of the VGAM and the brain may compete and venous congestion can be present, ${ }^{9}$ leading to chronic venous ischemia, delayed calcifications, and the so-called "pseudophlebitic" appearance of the cortical veins.

\section{CONCLUSIONS}

Decreased diameter of the superior sagittal sinus at presentation or jugular bulb narrowing or occlusion in 1- and 2-year-old children is associated with poor clinical outcome in patients with VGAM. The first may reflect a decrease of the cerebral blood flow due to cerebral arterial steal and intracranial hydrovenous disorders. At presentation, this finding could be considered a manifestation of cerebral blood flow deterioration and taken into consideration in the management decisions for patients with VGAM, even if the patients still have a good psychomotor development. The second may lead to pial venous congestion.

\section{APPENDIX \\ Bicêtre Outcome Score}

0. Death (D)

1. Severe neurologic symptoms, mental retardation of more than $20 \%$ (SNS), specialized school, and/or cardiac failure unstable despite treatment

2. Permanent minor neurologic symptoms, mental retardation of up to $20 \%$, nonpermanent neurologic symptoms under treatment (MNS), normal school with support, and/or cardiac failure stabilized with treatment

3. Transient neurologic symptoms, not treated (TNS), and/or asymptomatic cardiac overload under treatment

4. Minimal non-neurologic symptoms, not treated (MS), and/or asymptomatic enlargement of the cardiac silhouette

5. Normal (N).

\section{REFERENCES}

1. Alvarez H, Garcia Monaco R, Rodesch G, et al. Vein of galen aneurysmal malformations. Neuroimaging Clin N Am 2007;17:189-206 CrossRef Medline

2. Markowski J. Entwicklung der Sinus durae matris und der Hirnvenen des Menschen. Bulletin International de l'Acade'mie des Sci-

AJNR Am J Neuroradiol 37:1354-58 Jul 2016 www.ajnr.org

1357 
ences et des Lettres. Classe des Sciences Mathe' matiques et Naturelles. Serie B: Sciences Naturelles 1921;1-269

3. Lasjaunias P, Garcia-Monaco R, Rodesch G, et al. Deep venous drainage in great cerebral vein (vein of Galen) absence and malformations. Neuroradiology 1991;33:234-38 CrossRef Medline

4. Berenstein A, Masters LT, Nelson PK, et al. Transumbilical catheterization of cerebral arteries. Neurosurgery 1997;41:846-50 CrossRef Medline

5. Dahdah NS, Alesseh H, Dahms B, et al. Severe pulmonary hypertensive vascular disease in two newborns with aneurysmal vein of Galen. Pediatr Cardiol 2001;22:538-41 CrossRef Medline

6. Frawley GP, Dargaville PA, Mitchell PJ, et al. Clinical course and medical management of neonates with severe cardiac failure related to vein of Galen malformation. Arch Dis Child Fetal Neonatal Ed 2002;87:F144-49 CrossRef Medline

7. Johnston IH, Whittle IR, Besser M, et al. Vein of Galen malformation: diagnosis and management. Neurosurgery 1987;20: 747-58 CrossRef Medline

8. Jones BV, Ball WS, Tomsick TA, et al. Vein of Galen aneurysmal malformation: diagnosis and treatment of 13 children with extended clinical follow-up. AJNR Am J Neuroradiol 2002;23:1717-24 Medline

9. Lasjaunias PL, Berenstein A, terBrugge KG. Vein of Galen aneurysmal malformation. In: Lasjaunias PL, Berenstein A, terBrugge KG. Surgical Neuroangiography: Clinical and Interventional Aspects in Children. 2nd ed. Berlin: Springer-Verlag; 2006:105-226

10. Lasjaunias PL, Chng SM, Sachet M, et al. The management of vein of Galen aneurysmal malformations. Neurosurgery 2006;59(5 suppl 3): S184-94; discussion S3-13 Medline

11. Geibprasert S, Krings T, Armstrong D, et al. Predicting factors for the follow-up outcome and management decisions in vein of Galen aneurysmal malformations. Childs Nerv Syst 2010;26:35-46 CrossRef Medline

12. Li AH, Armstrong D, terBrugge KG. Endovascular treatment of vein of Galen aneurysmalmalformation:management strategy and 21-year experience in Toronto. J Neurosurg Pediatr 2011;7:3-10 CrossRef Medline

13. Raybaud CA, Hald JK, Strother CM, et al. Aneurysms of the vein of Galen: angiographic study and morphogenetic considerations [in French]. Neurochirurgie 1987;33:302-14 Medline 